\title{
MASTER Prompt and Follow-Up GRB Observations
}

\author{
Nataly Tyurina, ${ }^{1}$ Vladimir Lipunov, ${ }^{1}$ Victor Kornilov, ${ }^{1}$ \\ Evgeny Gorbovskoy, ${ }^{1}$ Nikolaj Shatskij, ${ }^{1}$ Dmitry Kuvshinov, ${ }^{1}$ Pavel Balanutsa, ${ }^{1}$ \\ Alexander Belinski, ${ }^{1}$ Vadim Krushinsky, ${ }^{2}$ Ivan Zalozhnyh, ${ }^{2}$ Andrey Tlatov, ${ }^{3}$ \\ Alexander Parkhomenko, ${ }^{3}$ Kirill Ivanov, ${ }^{4}$ Sergey Yazev, ${ }^{4}$ Peter Kortunov, ${ }^{1}$ \\ Anatoly Sankovich, ${ }^{1}$ Artem Kuznetsov, ${ }^{1}$ and Vladimir Yurkov ${ }^{5}$
}

\author{
${ }^{1}$ Sternberg Astronomical Institute, Moscow State University, 13, Univeristetskij pr-t, Moscow 119991, Russia \\ ${ }^{2}$ Ural State University, 51, Lenina pr-t, Ekaterinburg 620083, Russia \\ ${ }^{3}$ Kislovodsk Solar Station, P.O. Box 145, 100, Gagarina st., Kislovodsk 357700, Russia \\ ${ }^{4}$ Irkutsk State University, 1, Karl Marks ul., Irkutsk 664003, Russia \\ ${ }^{5}$ Blagoveschensk Pedagogical State University, 104, Lenina ul., Blagoveschensk 675000, Russia
}

Correspondence should be addressed to Nataly Tyurina, tyurina@sai.msu.ru

Received 3 November 2009; Revised 12 February 2010; Accepted 10 March 2010

Academic Editor: Alberto J. Castro-Tirado

Copyright ( 2010 Nataly Tyurina et al. This is an open access article distributed under the Creative Commons Attribution License, which permits unrestricted use, distribution, and reproduction in any medium, provided the original work is properly cited.

\begin{abstract}
We presented the results of last years GRB observations obtained on the MASTER robotic telescope, which is the only telescope of its kind in Russia. These results include 5 prompt observations of GRB in 2008 and 2009, follow-up observations of 15 other GRBs in 2008-2009, the first observations in different polarization angles of optical emission from the gamma-ray bursts GRB091020, and observations in different polarization angles for GRB091127 and GRB090820.
\end{abstract}

\section{Introduction}

The construction of robotic telescopes, which not only automatically acquire but also automatically process images and choose observing strategies, is a rather new and vigorously developing area in modern astronomy. MASTER (Mobile Astronomy System of Telescope Robots, http://observ .pereplet.ru), which is the first and still unique robotic telescope network in Russia, was started through the efforts of scientists at the Sternberg Astronomical Institute of Moscow State University and the Moscow "Optika" Association in 2002 and continues to be developed to the present MASTERNET [1-3].

MASTER is dedicated to the observation and detection of optical transients on time scales from few seconds to several days. The emphasis is on gamma-ray bursts (GRBs), the most powerful explosions in our Universe. A special program package for image reduction in real-time has been created, making it possible not only to carry out astrometry and photometry of a frame but also to recognize objects lacking in astronomical catalogs: supernovae, new asteroids, optical transients, and so forth. This software allowed us to discover several supernovae: SN2008gy, SN 2006ak, SN2005ee, and SN 2005bv [4].

We observed 20 error boxes of gamma-ray bursts in 20082009 and report the results obtained. There are a total of five prompt observations made with very-wide-field cameras (VWFC) during the past half-year after we mounted these cameras in Kislovodsk and Irkutsk in autumn 2009.

\section{MASTER System}

MASTER network currently consists of six facilities deployed near Moscow (MASTER-Moscow), Kislovodsk, Irkutsk, Ekaterinburg (MASTER-Ural system), and Blagovesh'ensk (MASTER-Amur one) [3]. We have distant observatories for only a year and a half, and for about five years, observations have been made only with the Moscow telescope facility located in a village near Domodedovo airport. Its location close to Moscow State University allowed us to design, test, and modify the robot system, repair eventual technical faults, and develop reduction software with no financial support from the state. But the negative side is that we had to observe under poor weather conditions near megalopolis (less than 


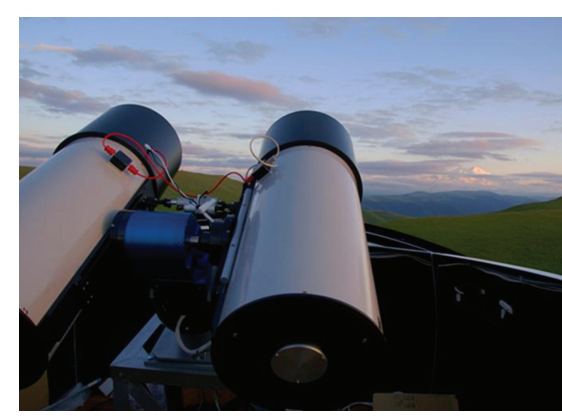

Figure 1: MASTER-II system in Kislovodsk observatory $(D=$ $400 \mathrm{~mm}$ ).

70 clear nights per year and most of them are summer white nights). However, we observed several tens of error boxes during five years (HETE and Swift epochs), and in most of the cases our observations were the earliest [2].

MASTER characteristics [3] are the closest to those of the American ROTSE-III network [5] (http://www.rotse.net/). MASTER differs in that its facilities have a larger field of view and several telescopes mounted on a single axis, allowing images to be taken at several different wavelengths simultaneously, as in the case of MASTER-Ural and MASTERKislovodsk observations.

Until 2008, most of the GRB observations were made with the MASTER-Moscow system, and we therefore report here its main properties.

The main telescope (with a $355 \mathrm{~mm}$ aperture) takes images in white light and serves as the main searching unit of the system. It has an Apogee Alta U16 (4096 × 4096 pixels) camera mounted on it, making it possible to obtain images in a six-square-degree field. In addition, MASTER has a verywide-field camera $\left(50^{\circ} \times 60^{\circ}\right)$ that covered the field of view of the HETE orbiting gamma-ray telescope and made it possible to obtain simultaneous observations with HETE down to a limiting magnitude of $9 \mathrm{~m}$ using a separate automated scheme. This wide-field facility is capable of searching for bright, transient objects exclusively.

MASTER-Kislovodsk allows us to take images of observed GRB in different wavelengths or in different polarizations simultaneously. This system has 2 main telescopes $(D=$ $400 \mathrm{~mm}$, [3], Figure 1) with filters. And we have very widefield cameras (Figure 2) on the Mountain Solar Station of the Pulkovo Main Astronomical Observatory, allowing us to continuously monitor a 420-square-degree sky field down to $13 \mathrm{~m}$ with five-second exposures. We have also installed such cameras in Irkutsk and Tunka systems. MASTER-Tunka has also a main telescope $(D=400 \mathrm{~mm})$. And now we have MASTER-Amur with a $200 \mathrm{~mm}$ aperture.

Tunka and Amur systems were mounted in October and November of 2009.

The Kislovodsk, Moscow, Ural, Irkutsk, and Amur facilities are linked via the Internet and are able to respond to the detection of uncatalogued objects (optical transients) within several tens of seconds (processing time included). The results of observations using the MASTER network will be reported separately.
All MASTER facilities can operate in a fully automated mode based on the ephemerides (sunset) provided there are satisfactory weather conditions: (the control computer is continuously attached to a weather sensor), the roofs (above the main mount and wide-field camera) are opened, the telescopes are pointed at bright stars, and pointing corrections are applied, and then, depending on the seeing, the facility either goes into a standby regime or begins a survey of the sky using a specialized, fully automated program.

Thus, observations are conducted in two modes: survey and "alert" (e.g., observations of the already obtained coordinates of the gamma-ray bursts). In the former case, the main telescope automatically takes three frames of an arbitrary region in succession with exposures ranging from 30 to $60 \mathrm{~s}$, moves to a neighboring region $2^{\circ}$ away, performs the same procedure, and so on, repeating a given set of three frames every 40-50 minutes. Such a procedure makes it possible to avoid artefacts in the data processing and locate moving objects.

The alert mode is supported by a continuous connection between the control computer and the GCN international gamma-ray burst (GRB) network (http://gcn.gsfc.nasa.gov/). After detection of a GRB by a space gamma-ray observatory (Swift, Konus, Fermi etc.), the telescope receives the coordinates of the burst region (coordinate error box), automatically points to this direction, takes an image of this region, reduces the image, and identifies all objects not present in the computer catalogs. If a GRB is detected during the day, its coordinates are included in the observing program for the next night.

A special program package for image reduction in realtime has been developed, which is capable not only of performing astrometric and photometric reduction of a frame but also of recognizing objects not contained in astronomical catalogs: supernovae, new asteroids, optical transients, and so forth. Since the start of its operation, the MASTER system has taken the images for a lot of GRB error boxes (see the results of 2002-2006 observations in $[1,2,4]$ ). In half of the cases, these observations were the first in the world.

\section{GRB Observations}

We have observations of 20 GRBs in 2008-2009. The results of our prompt and follow-up observations are listed in Table 1.

We observed 80 GRB error boxes from 2002 to 2009 (20 in 2008-2009, 5 in 2007, 17 in 2006, 17 in 2005, 13 in 2004, 7 in 2003, and 1 in 2002). They represent the only 9 cases with optical counterpart. The reasons are the following: at the beginning of our observations we have a lot of technical problems (like with the Internet in our Moscow village with MASTER-I), then for about 5 years we observed only from Moscow region (with very bad astroclimatic conditions), and most of GRB in the first years of observations (mostly HETE results) came only in our day, so we could observe error-boxes only several hours later. But although we have the earliest observations in Europe of GRB030329 and have 8 hours of continuous observations, we took the earliest images of GRB050824. 


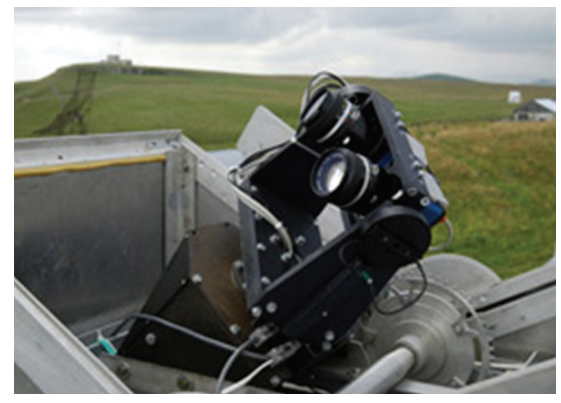

(a)

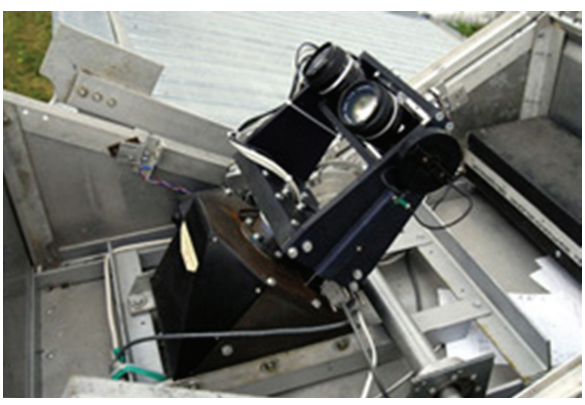

(b)

Figure 2: There are 4 MASTER VWFCs located at Kislovodsk observatory.

TABLE 1: GRB observations by MASTER system in 2008-2009.

\begin{tabular}{|c|c|c|}
\hline Burst & $\begin{array}{l}\text { Optical } \\
\text { limits, m }\end{array}$ & Comments (observatory, time after trigger information, exposures, circular number, etc.) \\
\hline GRB091130B & 15 & $\begin{array}{l}\text { MASTER-Amur, unfiltered image, } 10 \text {-s exposures. The time delay is } 40 \mathrm{~s} \text { after the notice time ( } 65 \mathrm{~s} \text { after the } \\
\text { GRB time). There is no OT brighter than } 15 \mathrm{~m} \text { at the Swift XRT position [6]. }\end{array}$ \\
\hline GRB091130 & 14.5 & $\begin{array}{l}\text { MASTER-Amur observations. } 23 \text { seconds after the notice time ( } 7 \text { min } 22 \mathrm{~s} \text { after the GRB time). We do not see } \\
\text { OT brighter than } 14 \text { mag in BAT error box [7]. }\end{array}$ \\
\hline GRB091127 & $\sim 14 \mathrm{mag}$ & $\begin{array}{l}\text { MASTER-II-Kislovodsk observed } 9 \text { seconds after notice time (91 s after the GRB time). OT images in both } \\
\text { polarizations [8]. }\end{array}$ \\
\hline GRB091020 & $17.9 \mathrm{R}$ & $\begin{array}{l}\text { MASTER-II-Kislovodsk has optical transient polarimetry observed OT } 3422 \mathrm{~s} \text { after the GRB time in R and } \\
\text { unfiltered. There are images in different polarization angles [9]. }\end{array}$ \\
\hline GRB090820 & 11 & $\begin{array}{l}\text { MASTER-Kislovodsk and Irkuts VWFC responded to Fermi trigger 272421498, } 9 \text { s after the GRB time. We took } \\
1300 \text { images with 5-second exposition in different polarization angles [10]. }\end{array}$ \\
\hline GRB090715B & 20 & $\begin{array}{l}\text { MASTER-Moscow observations, } 19 \mathrm{~s} \text { after the GCN notice time ( } 8 \mathrm{~m} 04 \mathrm{~s} \text { after the burst). } 160 \text { images with } \\
30 \text {-second expositions. No OT [11]. }\end{array}$ \\
\hline GRB090528B & $\begin{array}{l}\mathrm{R}>19.0 \\
\mathrm{~V}>18.1\end{array}$ & $\begin{array}{l}\text { MASTER-URAL, } 7 \text { hours after GRB, } 180 \text {-s exposures in R and V filters. Our images cover } 30 \% \text { last } 1-\sigma \text { Fermi } \\
\text { error box. No OT [12]. }\end{array}$ \\
\hline GRB090424 & $\sim 8, \sim 9$ & $\begin{array}{l}\text { Prompt observations by } 6 \text { MASTER VWFC in Kislovodsk and Irkutsk with common FOW }=6000 \mathrm{sq} \cdot \text { deg., } 1 \text {-s } \\
\text { exposures. Unfiltered images (close to V band). No OT [13]. }\end{array}$ \\
\hline GRB090408B & 17 & MASTER-URAL, R and V 60-s exposure, starting after sunset (87 min after trigger time). No OT [14]. \\
\hline GRB090328B & 9.1 & $\begin{array}{l}\text { Prompt observations at MASTER VWFC located at Irkutsk. 1-s exposures during night: before, during, and after } \\
\text { GRB. No OT [15]. }\end{array}$ \\
\hline GRB090320B & $\mathrm{V} \sim 9.0$ & $\begin{array}{l}\text { Prompt observation by MASTER VWFC located at Kislovodsk. We observed } \sim 80 \% 1-\sigma \text { Fermi error box with 1-s } \\
\text { exposure during all night: before, during, and after GRB time. No OT }[16] .\end{array}$ \\
\hline GRB090305B & $\mathrm{V} \sim 9.5$ & $\begin{array}{l}\text { Prompt observation by MASTER VWFC located at Kislovodsk observed } \sim 80 \% 1-\sigma \text { Fermi error box with 1-s } \\
\text { exposure } 7 \text { hours before, during, and } 1 \text { hour after GRB time [17]. }\end{array}$ \\
\hline GRB081215A & $\mathrm{V} \sim 11$ & $\begin{array}{l}\text { MASTER VWFC located at Kislovodsk observed Fermi trigger (SGR 0044+42) during all night with 5-s } \\
\text { expositions [18]. }\end{array}$ \\
\hline GRB081130B & $\mathrm{V} \sim 12$ & $\begin{array}{l}\text { Prompt observation. MASTER VWFC located at Kislovodsk observed Fermi trigger with 5-s exposures. No OT } \\
\text { [19]. }\end{array}$ \\
\hline GRB081110 & 19 & $\begin{array}{l}\text { MASTER-Moscow observed error box } 6 \text { hours } 42 \text { minutes after the GRB time. We have } 89 \text { images with limit for } \\
\text { coadded one up to } 19 \mathrm{~m}(\mathrm{~S} / \mathrm{N}=4) \text {. No OT [20]. }\end{array}$ \\
\hline GRB081102 & $\mathrm{V} \sim 13$ & $\begin{array}{l}2 \text { of MASTER VWFC at Kislovodsk observed this error box with 5-s exposures during all night. There are two } \\
\text { separated }(\sim 702 \mathrm{~m}) \text { mount with double cameras [21] }\end{array}$ \\
\hline GRB080822B & 18.8 & $\begin{array}{l}\text { MASTER-Moscow observed his gamma-ray burst } 18 \mathrm{~m} 43 \mathrm{~s} \text { after the GRB time with } 30 \text {-s exposures. Each image } \\
\text { has } 17 \mathrm{~m} \text {. Summary image }(25 \times 30 \mathrm{~s}) \text { has } 18.8 \mathrm{~m} \text {. The unfiltered images are calibrated relative to USNO A2.0 } \\
(0.8 \mathrm{R}+0.2 \mathrm{~B})[22] \text {. }\end{array}$ \\
\hline GRB080605 & $\mathrm{R} \sim 11.5$ & $\begin{array}{l}\text { MASTER VWFC at Kislovodsk observed it } 46 \mathrm{~s} \text { after the GRB time (12 s after the notice arrival time) with a } \\
\text { series of 5-s exposures. Unfiltered (close to V). No OT [23] }\end{array}$ \\
\hline GRB080319D & $\mathrm{V} \sim 11.5$ & $\begin{array}{l}\text { MASTER VWFC at Kislovodsk observed Swift-BAT trigger with a series of 5-s exposures starting } 92 \mathrm{~s} \text { after } \\
\text { notice arrival time ( } 708 \mathrm{~s} \text { after GRB time). Unfiltered (close to R, i.e., another camera). No OT [24]. }\end{array}$ \\
\hline GRB080205 & 19.5 & MASTER-Moscow observed it 481 minutes after the GRB time. No OT [25]. \\
\hline
\end{tabular}


TABLE 2: The result of the unfiltesred polarization.

\begin{tabular}{|c|c|c|c|c|c|}
\hline \multicolumn{3}{|c|}{ West-telescope } & \multicolumn{3}{|c|}{ East-telescope } \\
\hline GRB time, hour & OT mag & error & GRB time, hour & OT mag & error \\
\hline 1.605 & 18.65 & 0.10 & 1.605 & 18.60 & 0.06 \\
\hline 1.983 & 18.91 & 0.08 & 1.983 & 18.46 & 0.06 \\
\hline 2.286 & 19.04 & 0.08 & 2.286 & 19.14 & 0.09 \\
\hline 2.563 & 19.28 & 0.11 & 2.563 & 19.11 & 0.09 \\
\hline 2.840 & 19.36 & 0.09 & 2.840 & 19.56 & 0.10 \\
\hline 3.117 & 19.32 & 0.08 & 3.117 & 19.38 & 0.09 \\
\hline 3.395 & 19.35 & 0.06 & 3.395 & 19.70 & 0.17 \\
\hline 3.672 & 19.23 & 0.10 & 3.672 & 19.53 & 0.11 \\
\hline 3.949 & 20.07 & 0.16 & 3.949 & 19.92 & 0.16 \\
\hline 4.227 & 20.67 & 0.28 & 4.227 & 20.03 & 0.19 \\
\hline 4.505 & 20.07 & 0.22 & 4.505 & 19.99 & 0.16 \\
\hline
\end{tabular}

TABLE 3: The results of observations in 2007.

\begin{tabular}{|c|c|c|}
\hline Burst & $\begin{array}{l}\text { Optical } \\
\text { limits, } \mathrm{m}\end{array}$ & Comments (observatory, time after trigger information, exposures, circular number, etc.) \\
\hline GRB071122 & 16 & MASTER-Moscow observed it $151 \mathrm{~s}$ after the GRB time (61 s after notice time). No OT [35] \\
\hline GRB070810.8 & 14.8 & MASTER-Moscow observed it $125 \mathrm{~s}$ after the GRB time. No OT [36]. \\
\hline \multirow[t]{2}{*}{ GRB070224 } & 13 & $\begin{array}{l}\text { MASTER VWFC observed error box in Kislovodsk with series of 5-s exposures starting } 2 \mathrm{~s} \text { after notice arrival } \\
\text { time. Unfiltered (close to R). No OT. }\end{array}$ \\
\hline & 18 & MASTER-Moscow observed error box $51 \mathrm{~s}$ after notice arrivel time. Unfiltered. No OT [37]. \\
\hline GRB070223 & 13 & $\begin{array}{l}\text { MASTER VWFC observed error box in Kislovodsk 5-s exposures starting } 1 \text { s after notice arrival time. No OT. } \\
\text { Unfiltered (close to R) [38]. }\end{array}$ \\
\hline GRB070219 & 13.5 & $\begin{array}{l}\text { MASTER VWFC observed error box in Kislovodsk 5-s exposures starting } 76 \mathrm{~s} \text { after the GRB time and } 15 \mathrm{~s} \text { after } \\
\text { notice arrival time. No OT [39]. }\end{array}$ \\
\hline
\end{tabular}

We obtained a total of 5 prompt observations with the very-wide-field cameras in Irkutsk and Kislovodsk stations during the past half-year (after we had mounted several VWFC cameras there): GRB090424, GRB090328B, GRB090320B, GRB090305B, and GRB081130B. We found no optical candidate in the reduced images, and we give only the optical limits for GRBs. And we have several GRB's observations in different polarization angles (GRB091127, GRB091020, and GRB090820).

If we found no optical counterpart in the reduced images, we give only the optical limits for GRBs, that is, there is no optical transient brighter than this limit.

Kislovodsk and Irkutsk VWFC had different cameras during 2008, and that is why we give V-and R-band data in the second column of the table. We have unfiltered images from these cameras, but they are the closest to $\mathrm{V}$ and $\mathrm{R}$ bands, and we calibrated them using Tycho catalogue [26]. We also calculate the ratio of optical to gamma fluence [26] for GRBs.

We detected several interesting GRBs during this two years, and we now briefly discuss them.

3.1. GRB091127. MASTER-II robotic telescope located at Kislovodsk has early optical transient polarimetry of this burst. System responded to the GRB 091027 [27] 9 seconds after notice time and $91 \mathrm{~s}$ after the GRB time at very large zenit distance ( $\sim 4$ degrees up to horizon). We have bright optical counterpart ( 14 mag, 91-111 seconds after the GRB time) at Liverpool position [28] in both polarizations. We have 20 images with synchronous exposition in two polarizations before GRB setting with growing exposition from 20 to 160 seconds. And we had possible brightening around 2 minutes (after GRB Time).

3.2. GRB091020. MASTER-II robotic telescope located at Kislovodsk was pointed to the GRB 091020 [29] by the Internet $3422 \mathrm{~s}$ after the GRB time. The large delay was caused with technical problems on site [30].

We have 2 first images in different polarization angles (East telescope and West one, Figure 3).

The first 7 images was taken in R-band. The exposure of each image is $180 \mathrm{~s}$.

The result of the unfiltered polarization is in Table 1.

Using all our polarized magnitudes, we find that the light curve is very well described by a single power-law decay with alpha $=1.2 \pm 0.1$, that is in good agreement with the following observations [31].

The light curve of our observations is available at http:// observ.pereplet.ru/images/GRB091020/grb.html. 


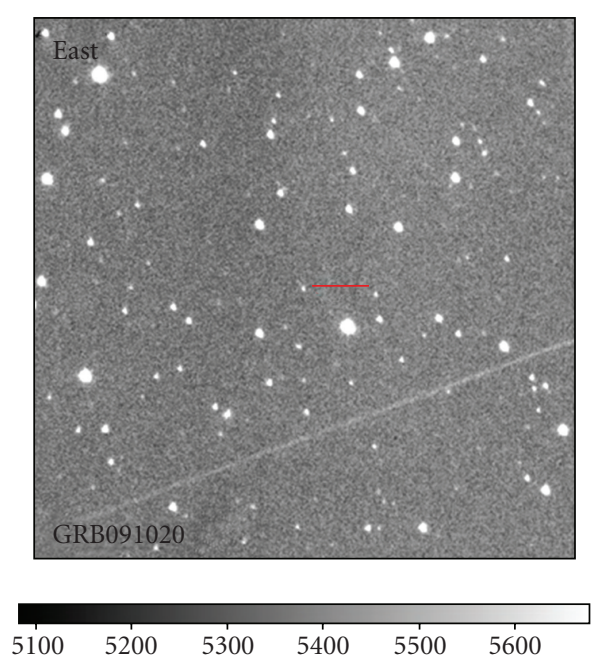

(a)
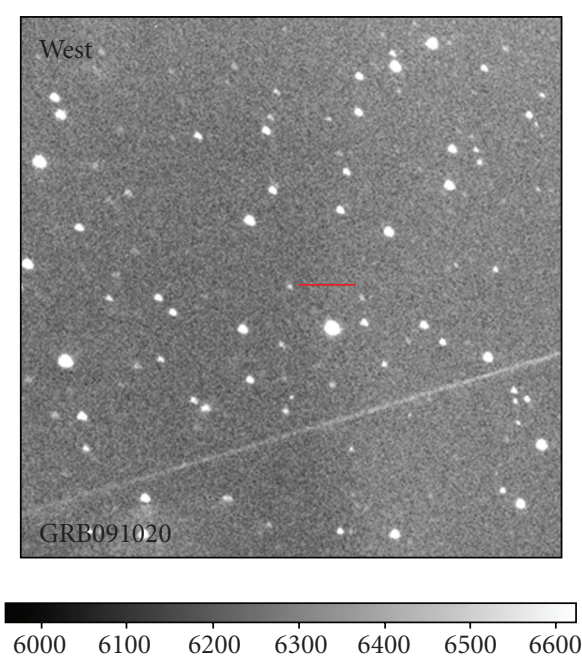

(b)

Figure 3: GRB091020 images.

3.3. GRB 090820. We had MASTER-Net optical polarization observations.

There are 6 MASTER very-wide-field cameras located at Kislovodsk and Irkutsk with common FOW $=2000+$ 1680 square degrees observed this bright Fermi trigger [32].

MASTER VWFC, located in Kislovodsk, has produced images beginning at $9 \mathrm{~s}$ after the GRB time (first alert notice). We have 1300 images with 5-second exposition in different polarization angles. Individual images have limiting magnitudes $\sim 11$. Our unfiltered images are calibrated relative to Tycho stars (V) and very close to V-band.

3.4. GRB090715B. MASTER robotic telescope located at Moscow $(D=355 \mathrm{~mm}$, Apogee Alta U16) responded to the GRB 090715B (Swift trigger, [33]). We have images beginning at $19 \mathrm{~s}$ after the GCN notice time. An automated response took the first image at 21:11:18 UT, $8 \mathrm{~m} 04 \mathrm{~s}$ after the burst, under summer Moscow sky.

We have 160 images with 30-second exposition each. These unfiltered images are calibrated relative to USNO 08. $\mathrm{R}+0.2 \mathrm{~B}$ (Usno A2.0). Individual images have limiting magnitudes ranging from 17.8 to 17.0 .

No optical transient was found brighter 20.0.

3.5. GRB090305B-MASTER-Net Prompt Optical Short Burst Observations. We observed this burst by 6 MASTER verywide-field cameras located at Kislovodsk and Irkutsk with common FOW $=6000$ square degrees. One of the four MASTER very-wide-field Cameras located at Kislovodsk ( $D=50 \mathrm{~mm}, 4 \times 1000$ square degrees, $11 \mathrm{Mpx}$ ) has observed $\sim 80 \%$ 1-sigma Fermi error box [34] with 1-s exposure during all night: 7 hours before, during, and 1 hour after GRB time without time gap between images.

Our unfiltered images were calibrated relative to Tycho stars (V). The magnitude limit of each image is $\sim 9.5 \mathrm{~m}$ at the edge of FOW. We do not detect OT inside the part of the error box brighter than 9.2 mag before and after trigger time. So we conclude (in the case that GRB is inside our FOW) that the optical fluence during the short 2- s GRBburst is limited by $\sim \leq 1 \times 10^{-8} \mathrm{erg} / \mathrm{cm}^{2}$ (excluding possible host galaxy absorption).

3.6. Summary. There were 5 observations in 2007 with no optical counterpart, because we observed them by Moscow telescope and only VWFC in Kislovodsk, that was just mounted and worked in a half-tested mode.

\section{Conclusions}

We presented the results of last years GRB observations obtained on the MASTER robotic telescope, which is the only telescope of its kind in Russia. These results include 5 prompt observations of GRB in 2008 and 2009, follow-up observations of 15 other GRBs in 2008-2009, the first observations in different polarization angles of optical emission from the gamma-ray bursts GRB091020, and observations in different polarization angles for GRB091127 and GRB090820.

Our experience of two years of operation of the MASTER wide-field robotic telescope has demonstrated its unique capabilities. We hope that new MASTER telescopes at Kislovodsk and Ural stations and also new Irkutsk and Blagoveschwnsk systems will justify our hopes. We have 3 optical counterparts in the last half of 2009. When such systems will be installed at suitable sites at various hour angles across Russia, they would provide unique information via continuous monitoring of both the near and distant cosmos.

\section{Acknowledgment}

The authors thank the General Director of the "OPTIKA" Association S.M. Bodrov for providing the MASTER project with necessary expensive equipment. 


\section{References}

[1] V. M. Lipunov, A. V. Krylov, V. G. Kornilov, et al., "MASTER: the mobile astronomical system of telescope-robots," Astronomische Nachrichten, vol. 325, no. 6-8, pp. 580-582, 2004.

[2] V. M. Lipunov, V. G. Kornilov, A. V. Krylov, et al., "The master mobile astronomical system. Optical observations of gammaray bursts," Astrophysics, vol. 48, no. 3, pp. 389-399, 2005.

[3] V. M. Lipunov, V. G. Kornilov, E. Gorbovskoyv, et al., "Master robotic net," Advances in Astronomy, vol. 2010, Article ID 349171, 6 pages, 2010.

[4] V. Lipunov, V. G. Kornilov, A. V. Krylov, et al., "Optical observations of gamma-ray bursts, the discovery of supernovae 2005bv, 2005ee, and 2006ak, and searches for transients using the "MASTER" robotic telescope," Astronomy Reports, vol. 51, no. 12, pp. 1004-1025, 2007.

[5] S. A. Yost, F. Aharonian, C. W. Akerlof, et al., "Status of the ROTSE-III telescope network," Astronomische Nachrichten, vol. 327, no. 8, pp. 803-805, 2006.

[6] S. Sergienko, V. Yurkov, E. Gorbovskoy, et al., "GRB 091130B: MASTER observations,” GCN Circular, no. 10216, 2009.

[7] S. Sergienko, V. Yurkov, E. Gorbovskoy, et al., "GRB 091130: MASTER observations,” GCN Circular, no. 10213, 2009.

[8] A. Belinski, E. Gorbovskoy, V. M. Lipunov, et al., "GGRB 091127: MASTER early optical transient polarimetry," GCN Circular, no. 10203, 2009.

[9] E. Gorbovskoy, V. Lipunov, V. Kornilov, et al., "GRB 091020: MASTER-Net optical polarization observations," GCN Circularno. 10231, 2009.

[10] E. Gorbovskoy, V. Lipunov, V. Kornilov, et al., "GRB 090820: MASTER-Net optical polarization observations," GCN Circular, no. 9830, 2009.

[11] D. Kuvshinov, V. Lipunov, V. Kornilov, et al., "GRB 090715B: MASTER optical limit," GCN Circular, no. 9681, 2009.

[12] V. Krushinski, I. Zalognikh, J. Popov, et al., "GRB 090528B: MASTER-II Kourovka optical limit,” GCN Circular, no. 9468, 2009.

[13] E. Gorbovskoy, V. Lipunov, V. Kornilov, et al., "GRB 090424: MASTER-Net prompt optical limit," GCN Circular, no. 9252, 2009.

[14] V. Krushinski, I. Zalognikh, T. Kopytova, et al., "GRB 090408B: MASTER II-Kourovka optical observations," GCN Circular, no. $9111,2009$.

[15] K. Ivanov, S. Yazev, E. Gorbovskoy, et al., "GRB 090328B: MASTER-Irkutsk prompt optical short burst observations," GCN Circular, no. 9065 , 2009.

[16] E. Gorbovskoy, V. Lipunov, V. Kornilov, et al., "GRB 090320B: MASTER-Net prompt optical observations," GCN Circular, no. $9038,2009$.

[17] E. Gorbovskoy, V. Lipunov, V. Kornilov, et al., "GRB090305B: MASTER-net prompt optical short burst observations," GCN Circular, no. $9004,2009$.

[18] E. Gorbovskoy, V. Lipunov, V. Kornilov, et al., "Fermi Trig Num 251059717 = SGR 0044+42: MASTER prompt optical observations," GCN Circular, no. 8674, 2008.

[19] E. Gorbovskoy, V. Lipunov, V. Kornilov, et al., "GRB 081130B: MASTER prompt optical observations," GCN Circular, no. 8597, 2008.

[20] D. Kuvshinov, E. Gorbovskoy, V. Lipunov, et al., "GRB 081110: MASTER: first optical observations Fermi GRB," GCN Circular, no. 8518, 2008.
[21] E. Gorbovskoy, V. Lipunov, V. Kornilov, et al., "GRB 081102: MASTER refind and final results," GCN Circular, no. 8516, 2008.

[22] D. Kuvshinov, V. Lipunov, V. Kornilov, et al., "GRB 080822B: MASTER optical observation,” GCN Circular, no. 8123, 2008.

[23] D. Kuvshinov, V. Lipunov, V. Kornilov, et al., "GRB 080605: MASTER VWF 46 s AT observation,” GCN Circular, no. 7836, 2008.

[24] V. Lipunov, V. Kornilov, D. Kuvshinov, et al., "GRB 080319D: MASTER-VWF-Kislovodsk optical observation," GCN Circular, no. 7455, 2008.

[25] D. Kuvshinov, V. Lipunov, V. Kornilov, et al., "GRB 080205: MASTER optical observation,” GCN Circular, no. 7261, 2008.

[26] E. Gorbovskoy, et al., Advances in Astronomy. In press.

[27] E. Troja, S. Barthelmy, J. Wren, W. Baumgartner, et al., "GRB 091127: Swift detection of a burst," GCN Circular, no. 10191, 2009.

[28] R. Smith, S. Kobayashi, C. Guidorzi, et al., "GRB 091127: Liverpool Telescope afterglow candidate," GCN Circular, no. 10192, 2009.

[29] J. Racusin, S. Barthelmy, A. Beardmore, et al., "GRB 091020: Swift detection of a burst with an optical afterglow," GCN Circular, no. 10048, 2009.

[30] E. Gorbovskoy, D. Kuvshinov, V. Lipunov, et al., "GRB 091020: MASTER optical transient polarimetry," GCN Circular, no. 10052, 2009.

[31] D. Kann, U. Laux, M. Roeder, and H. Meusinger, "GRB 091020: early TLS multicolor detections, red afterglow," GCN Circular, no. 10076, 2009.

[32] V. Connaughton, "GRB 090820: Fermi GBM Detection of a bright burst," GCN Circular, no. 9829, 2009.

[33] L. Vetere, A. P. Beardmore, N. Gehrels, et al., "GRB 090715B: Swift detection of a burst with a possible optical counterpart," GCN Circular, no. 9668, 2009.

[34] C. Wilson-Hodge and Fermi GBM Team, "GRB090305B: Fermi GBM detectionS,” GCN Circular, no. 8972, 2009.

[35] V. Lipunov, V. Kornilov, D. Kuvshinov, et al., "GRB 071122: MASTER optical observation,” GCN Circular, no. 7129, 2008.

[36] V. Lipunov, V. Kornilov, D. Kuvshinov, et al., "GRB 070810: MASTER optical observation,” GCN Circular, no. 6752, 2008.

[37] V. Lipunov, V. Kornilov, D. Kuvshinov, et al., "GRB 070224: MASTER-Net refind analysis," GCN Circularno. 6140, 2008.

[38] V. Lipunov, V. Kornilov, N. Shatskiy, et al., "GRB070223: MASTER-VWF-Kislovodsk observations," GCN Circular, no. 6131, 2008.

[39] V. Lipunov, V. Kornilov, N. Shatskiy, et al., "GRB070219: MASTER-VWF-Kislovodsk observations," GCN Circular, no. 6113, 2008. 

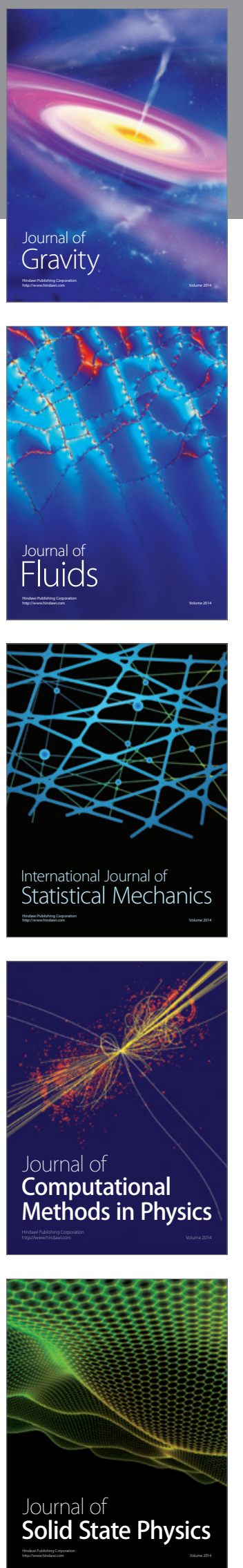

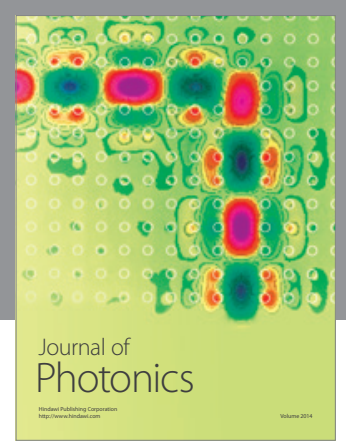

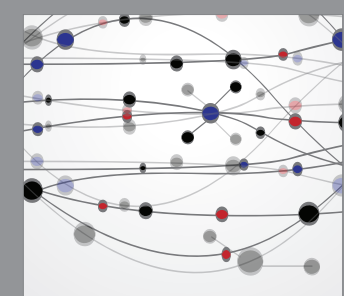

The Scientific World Journal
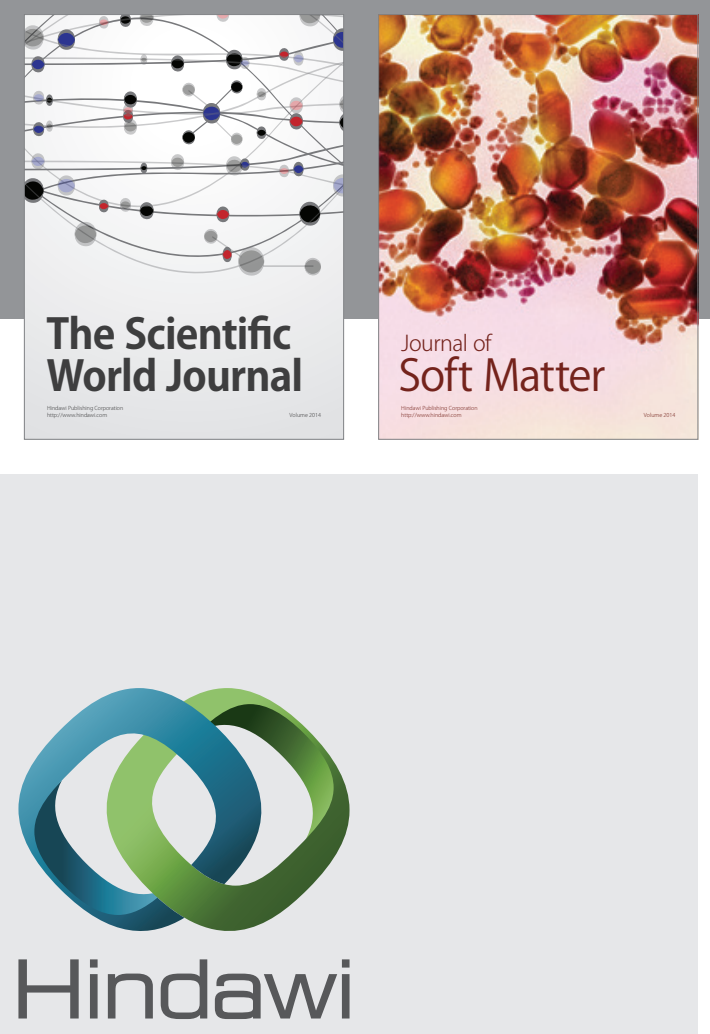

Submit your manuscripts at

http://www.hindawi.com
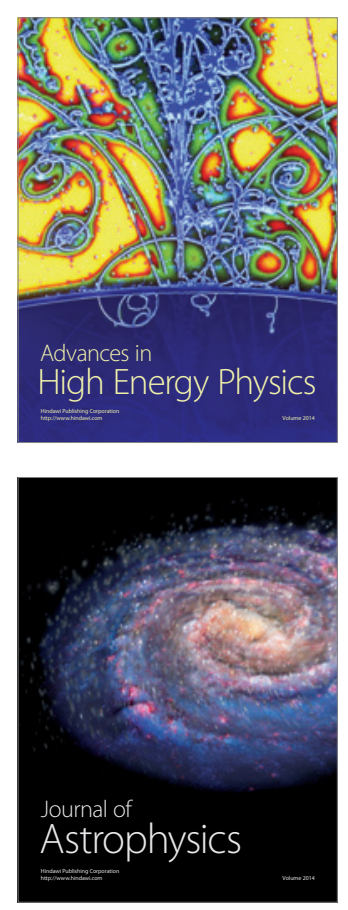
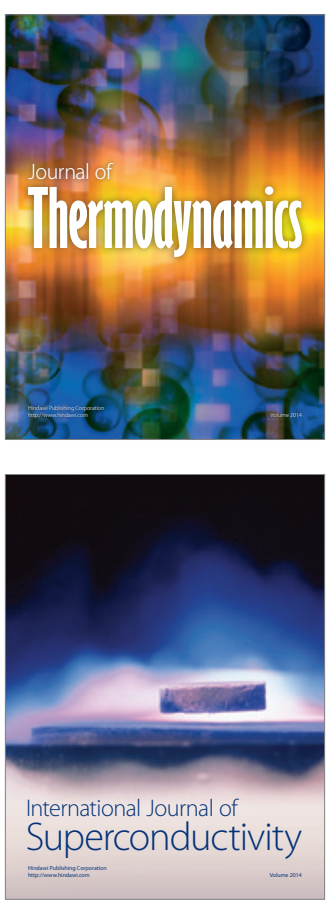
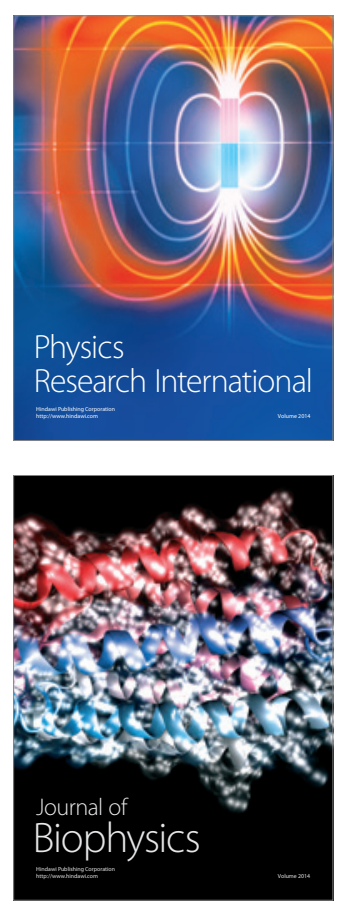
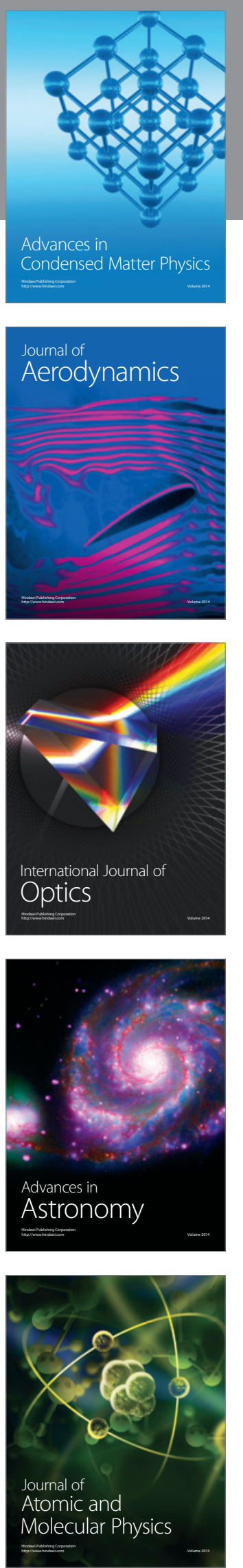